\title{
THE ADVANCED CAMERA FOR SURVEYS GENERAL CATALOG: STRUCTURAL PARAMETERS FOR APPROXIMATELY HALF A MILLION GALAXIES
}

\author{
Roger L. Griffith ${ }^{1}$, Michael C. Cooper ${ }^{2,14}$, Jeffrey A. Newman ${ }^{3}$, Leonidas A. Moustakas ${ }^{4}$, Daniel Stern $^{4}$, \\ Julia M. Comerford ${ }^{5}$, Marc Davis ${ }^{6}$, Jennifer M. Lotz $^{7}$, Marco Barden ${ }^{8}$, Christopher J. Conselice ${ }^{9}$, Peter L. Capak ${ }^{10}$, \\ S. M. Faber ${ }^{11}$, J. Davy KirkPatrick ${ }^{1}$, Anton M. KoeKemoer ${ }^{7}$, David C. Koo $^{11}$, Kai G. Noeske ${ }^{12,15}$, Nick Scoville ${ }^{10}$, \\ Kartik Sheth $^{10}$, Patrick Shopbell ${ }^{10}$, Christopher N. A. Willmer ${ }^{13}$, and Benjamin Weiner ${ }^{13}$ \\ ${ }^{1}$ Infrared Processing and Analysis Center, California Institute of Technology, Pasadena, CA 91125, USA \\ ${ }^{2}$ Center for Galaxy Evolution, Department of Physics and Astronomy, University of California, Irvine, 4129 Frederick Reines Hall, Irvine, CA 92697, USA \\ ${ }^{3}$ Pittsburgh Particle Physics, Astrophysics, and Cosmology Center, Department of Physics and Astronomy, University of Pittsburgh, Pittsburgh, PA 15260, USA \\ ${ }^{4}$ Jet Propulsion Laboratory, California Institute of Technology, 4800 Oak Grove Dr., Pasadena, CA 91109, USA \\ ${ }^{5}$ Astronomy Department, University of Texas at Austin, Austin, TX 78712, USA \\ ${ }^{6}$ Department of Astronomy, University of California, Berkeley, Hearst Field Annex B, Berkeley, CA 94720, USA \\ ${ }^{7}$ Space Telescope Science Institute, 3700 San Martin Dr., Baltimore, MD 21218, USA \\ ${ }^{8}$ Institute of Astro- and Particle Physics, University of Innsbruck, Technikerstr. 25, 6020 Innsbruck, Austria \\ ${ }^{9}$ School of Physics \& Astronomy, University of Nottingham, Nottingham, UK \\ ${ }^{10}$ Spitzer Science Centre, 314-6 California Institute of Technology, 1200 E. California Blvd., Pasadena, CA 91125, USA \\ ${ }^{11} \mathrm{UCO} /$ Lick Observatory, University of California, CA, USA \\ ${ }^{12}$ Harvard-Smithsonian Center for Astrophysics, Cambridge, MA, USA \\ ${ }^{13}$ Steward Observatory, University of Arizona, 933 North Cherry Avenue, Tucson, AZ 85721, USA \\ Received 2012 February 10; accepted 2012 March 7; published 2012 May 4
}

\begin{abstract}
We present the Advanced Camera for Surveys General Catalog (ACS-GC), a photometric and morphological database using publicly available data obtained with the Advanced Camera for Surveys (ACS) instrument on the Hubble Space Telescope. The goal of the ACS-GC database is to provide a large statistical sample of galaxies with reliable structural and distance measurements to probe the evolution of galaxies over a wide range of look-back times. The ACS-GC includes approximately 470,000 astronomical sources (stars + galaxies) derived from the AEGIS, COSMOS, GEMS, and GOODS surveys. GalapaGos was used to construct photometric (SEXTRACTOR) and morphological (GALFIT) catalogs. The analysis assumes a single Sérsic model for each object to derive quantitative structural parameters. We include publicly available redshifts from the DEEP2, COMBO-17, TKRS, PEARS, ACES, CFHTLS, and zCOSMOS surveys to supply redshifts (spectroscopic and photometric) for a considerable fraction $(\sim 74 \%)$ of the imaging sample. The ACS-GC includes color postage stamps, GALFIT residual images, and photometry, structural parameters, and redshifts combined into a single catalog.
\end{abstract}

Key words: catalogs - galaxies: evolution - galaxies: photometry - galaxies: structure - surveys

Online-only material: color figures, Supplemental data file (tar.gz)

\section{INTRODUCTION}

The detailed study of galaxy evolution began with the pioneering work of Edwin Hubble at Mt. Wilson Observatory in the 1920s. Hubble pioneered the investigation of galaxy properties by classifying galaxies according to their morphological structure, leading to the Hubble sequence of galaxies (Hubble 1926). In the local universe, the Hubble sequence is well defined and widely used; however, as one goes back in distance and cosmic time, morphological classification becomes an increasingly difficult problem. The advent and rapid growth of CCD technology within the past 30 years has allowed astronomers to image and catalog galaxies that were inaccessible in previous studies. In order to build a deep, comprehensive and coherent theory on galaxy evolution, complete samples of galaxies spanning a wide range of redshifts and look-back times are essential.

Within the past 10 years several large Hubble Space Telescope (HST) imaging surveys have been undertaken by various groups, each with their own goals and strategies, all utilizing the Advanced Camera for Survey's (ACS) high-resolution wide field camera (WFC; Clampin et al. 2002). The All-wavelength Extended Groth strip International Survey (AEGIS; Davis et al.

\footnotetext{
${ }^{14}$ Hubble Fellow.

${ }^{15}$ Keck Foundation Fellow.
}

2007) is centered on the Extended Groth Strip (EGS) and is one of four fields targeted by the DEEP2 Galaxy Redshift Survey (Davis et al. 2003; Newman et al. 2012) for extensive spectroscopic follow-up. The Cosmological Evolutionary Survey (COSMOS; Scoville et al. 2007) was designed around the large single-band $H S T$ survey with extensive follow-up spectroscopy from the zCOSMOS redshift survey (Lilly et al. 2009). A major aim of the DEEP2, AEGIS, and COSMOS surveys is to study galaxy evolution in the context of large-scale structure. The Great Observatories Origins Deep Survey (GOODS) (Dickinson et al. 2003; Giavalisco et al. 2004) was designed to be one of the deepest $H S T$ imaging campaigns to date; with its small area but deep imaging, it was designed to probe galaxy evolution down to the faintest galaxies detectable. The Galaxy Evolution from Morphology and SEDs (GEMS) survey (Caldwell et al. 2008) was designed to study galaxy evolution using multi-wavelength data to construct spectral energy distributions (SEDs) and measure morphologies.

The Advanced Camera for Surveys General Catalog (ACSGC) unifies the largest HST ACS imaging surveys into a single, homogeneously analyzed data set. We used the Galaxy Analysis over Large Areas: Parameter Assessment by Galfitting Objects from SExtractor (GalaPagos) code (Häußler et al. 2011), which incorporates both GALFIT (Peng et al. 2002) and SEXTRACTOR (Bertin \& Arnouts 1996) to construct photometric 
Table 1

ACS-GC Survey Fields

\begin{tabular}{lccccc}
\hline \hline Survey & R.A. & Decl. & Area & Filters & Pixel Scale \\
\hline & $(\mathrm{J} 2000)$ & $(\mathrm{J} 2000)$ & $\left(\mathrm{deg}^{2}\right)$ & & $\left(^{\prime \prime} \mathrm{pixel}^{-1}\right)$ \\
\hline AEGIS & $14: 17: 00$ & $+52: 30: 00$ & 0.197 & F606W and F814W & 0.03 \\
GOODS-N & $12: 36: 55$ & $+62: 14: 15$ & 0.07 & F606W and F775W & 0.03 \\
COSMOS & $10: 00: 28$ & $+02: 12: 21$ & 1.8 & F814W & 0.05 \\
GEMS & $03: 32: 25$ & $-27: 48: 50$ & 0.21 & F606W and F850LP & 0.03 \\
GOODS-S & $03: 32: 30$ & $-27: 48: 20$ & 0.07 & F606W and F850LP & 0.03 \\
\hline
\end{tabular}

and morphological catalogs derived from the HST ACS imaging. We provide additional derived data products (e.g., color images, atlas images, GALFIT residual images, and ACS FITS image cutouts) for every source in the catalog. We also provide redshifts collated from the various redshift surveys that accompany the imaging data for a large fraction of the sources. The main goal of the ACS-GC data set is to provide a large statistical sample of galaxies with reliable structural and distance measurements (for a subsample) to probe the evolution of galaxies over a wide range of look-back times. This data set can be utilized for various purposes, for example, these data have been used by Georgakakis et al. (2009) to study the host galaxy morphologies of X-ray-selected active galactic nuclei (AGNs) in the AEGIS, GOODS-S, and GEMS surveys. Comerford et al. (2009) report the serendipitous discovery of a dual AGN in the COSMOS field. Pierce et al. (2010) study the effects an AGN has on host galaxy colors and morphological measurements. Griffith \& Stern (2010) study the morphological distributions of AGNs selected using X-ray, radio, and IR imaging from the COSMOS survey. Masters et al. (2011) study the morphology of galaxies in the Baryon Oscillation Spectroscopic Survey. Cooper et al. (2012) study the impact of environment on the size evolution of massive early-type galaxies at intermediate redshift. Holden et al. (2012) study the evolution in the intrinsic shape distribution of early-type galaxies from $z \sim 1$ to $z \sim 0$. Welikala $\&$ Kneib (2012) study color gradients in galaxies out to $z \sim 3$.

There are a handful of standard galaxy properties that are commonly quantified, such as apparent magnitude, color, morphology/shape, redshift/distance, size, velocity dispersion, and metallicity. These can all be used to gain insight into the formation history and evolution of galaxies. Understanding how these properties change and evolve with redshift/time is integral in our construction of galaxy evolutionary models and scenarios. The combination of high-resolution, deep optical imaging and redshift measurements along with the structural parameters provided by the ACS-GC makes it a powerful data set that can be used to study the evolution of galaxy structures over cosmic times. In Section 2, we describe the imaging and redshift surveys used to construct the ACS-GC. We describe the redshift completeness and reliability in Section 3. We give a brief description of the quantitative analysis in Section 4. In Section 5, we describe properties of the ACS-GC catalog, including the naming conventions and auxiliary data products. We summarize this work in Section 6. All magnitudes are given in the AB magnitude system.

\section{THE REDSHIFT AND IMAGING DATA}

In this section, we describe the HST ACS imaging used to construct the ACS-GC data set and give basic descriptions of the imaging properties. We also summarize the available redshifts acquired from the various surveys, both spectroscopic and photometric. We summarize the ACS imaging data in Table 1, giving central coordinates for the surveys, survey size, filters, and pixel scales. Table 3 summarizes basic catalog statistics, giving number counts in the respective ACS filters and the total number of spectroscopic (split by quality) and photometric redshifts.

\subsection{The AEGIS Survey}

\subsubsection{Imaging}

The AEGIS (Davis et al. 2007) is a large collaborative effort designed to provide one of the largest and deepest panchromatic data sets currently available. The region studied is centered on the EGS $\left(\alpha=14^{\mathrm{h}} 17^{\mathrm{m}}, \delta=52^{\circ} 30^{\prime}\right)$, a region with deep observations covering all major wavebands from X-ray to radio. The HST ACS imaging in the EGS field is composed of 63 pointings using both the F606W and F814W filters, with exposure times of 2260 and 2100 s, respectively, per pointing. The imaging covers a total area of $\sim 710 \mathrm{arcmin}^{2}$. Our analysis is based on images produced by the STSDAS multidrizzle package (Koekemoer et al. 2002), and the final images have a pixel scale of 0.03 pixel $^{-1}$. For an extended object the $5 \sigma$ limiting magnitudes are $\mathrm{F} 606 \mathrm{~W}=26.2(\mathrm{AB})$ and $\mathrm{F} 814 \mathrm{~W}=25.6(\mathrm{AB})$.

\subsubsection{Redshifts}

For the AEGIS survey we provide a total of 5765 spectroscopic redshifts, of which 4244 are high-quality redshifts $\left(z_{q} \geqslant 3\right)$ from the DEEP2 galaxy redshift survey data release 3 (DR3; Davis et al. 2007). DEEP2 targets were selected for spectroscopy from the CFHT $12 \mathrm{~K}$ BRI imaging described in Davis et al. (2007). Eligible DEEP2 targets have $18.5 \leqslant R \leqslant 24.1$ and surface brightness $\mu_{R}=R+2.5 \log A<26.5$, where $A$ is the area of the aperture (in square arcseconds) used to measure the CFHT $12 \mathrm{~K} R$-band magnitude. The DEEP2 catalog provides a quality metric $\left(z_{q}\right)$ ranging from 1 for the lowest quality to 4 for the highest quality redshifts. Two significant features must match the spectral templates for a secure redshift (quality $z_{q} \geqslant 3$ ); note that a resolved [O II] $\lambda 3727$ doublet is counted as two features. The median redshift for the sample is 0.74 . Galaxies at $z>1.4$ generally lack strong features in the DEEP2 spectral window; these objects compose the bulk of the DEEP2 redshift failures. Ongoing spectroscopic efforts in the field as part of the DEEP3 Galaxy Redshift Survey (Cooper et al. 2011a; Cooper et al. 2011b) will significantly increase the completeness within the $H S T /$ ACS footprint.

We also provide 43,796 photometric redshifts as described in Coupon et al. (2009). Comparing with galaxy spectroscopic redshifts, in the wide fields, they find a photometric redshift dispersion of $0.037-0.039$ and an outlier rate of $3 \%-4 \%$ at $i_{\mathrm{AB}}^{\prime}<22.5$. Beyond $i_{\mathrm{AB}}^{\prime}=22.5$ the number of outliers rises from $5 \%$ to $10 \%$ at $i_{\mathrm{AB}}^{\prime}<23$ and $i_{\mathrm{AB}}^{\prime}<24$, respectively. The 
redshift range $0.2<z \leqslant 1.5$ is the most suitable since this redshift range is better constrained by the filters used.

\subsection{The GOODS Survey}

\subsubsection{Imaging}

The GOODS survey (Dickinson et al. 2003; Giavalisco et al. 2004) was designed to be a deep multi-wavelength data set with which to study the formation and evolution of galaxies. The GOODS survey targeted two separate fields, the Hubble Deep-Field North (HDF-N) (now referred to as GOODS-N) and the Chandra Deep-Field South (CDF-S) (now referred to as GOODS-S). The HST ACS imaging was carried out in four broad, non-overlapping filters, $\mathrm{F} 435 \mathrm{~W}(B), \mathrm{F} 606 \mathrm{~W}(V)$, $\mathrm{F} 775 \mathrm{~W}(i)$, and $\mathrm{F} 850 \mathrm{LP}(z)$. While the $\mathrm{F} 435 \mathrm{~W}$ images were all acquired at the beginning of the survey, the F606W, F775W, and F850LP were carried out in five epochs. The mean exposure time at each epoch was 1050, 1050, and $2100 \mathrm{~s}$ in the F606W, F775W, and F850LP bands, respectively. The imaging comprises 17 HST pointings in GOODS-N and 15 in GOODS-S. Our analysis is based on images produced by the STSDAS multidrizzle package, and the final images have a pixel scale of 0.03 pixel $^{-1}$. We restrict our analysis to the $\mathrm{F} 606 \mathrm{~W}$ and $\mathrm{F} 775 \mathrm{~W}$ imaging in GOODS-N and the F606W and F850LP imaging in GOODS-S. For GOODS-S we analyzed the F850LP filter in order to combine directly with the GEMS F850LP imaging. The ACS imaging covers a total area of $\sim 320 \operatorname{arcmin}^{2}$ (e.g., $160 \mathrm{arcmin}^{2}$ per field). The $5 \sigma$ limiting magnitudes for an extended source are $\mathrm{F} 606 \mathrm{~W}=25.7$ and $\mathrm{F} 775 \mathrm{~W}=25.0$.

\subsubsection{Redshifts}

For the GOODS-N survey we provide 2854 spectroscopic redshifts from various sources, of which 1347 are high-quality redshifts $\left(z_{q} \geqslant 3\right)$. To keep track and organize the different sources for spectroscopic redshifts, we provide a parameter called Z_ORIGIN. For $z_{-}$origin equal to GOODS-N-ALL, refer to Wirth et al. (2004) and Cowie et al. (2004); for the remainder of the spectroscopic redshifts, refer to Barger et al. (2008).

We provide 6278 photometric redshifts as described in Bundy et al. (2009). Compared to spectroscopic redshifts, the photometric redshift outliers (defined by $\left|z_{\mathrm{spec}}-z_{\text {phot }}\right|>1$ ) account for $4 \%$ of the redshift estimates, with $\sigma_{|\Delta z| /\left(1+z_{\text {spec }}\right)} \approx 0.1$ when outliers are excluded.

\subsection{The COSMOS Survey}

\subsubsection{Imaging}

The Cosmological Evolution Survey (COSMOS; Scoville et al. 2007) was designed to thoroughly probe the evolution of galaxies, AGNs, and dark matter in the context of their environment, and to sample the full dynamic range of largescale structure from voids to very massive clusters. COSMOS acquired the largest contiguous HST ACS imaging survey to date, covering $\sim 1.8 \mathrm{deg}^{2}$ in the F814W filter. The original HST imaging consisted of 590 pointings. We use the publicly available mosaics described in Koekemoer et al. (2007). The total mean exposure time for each pointing is $2028 \mathrm{~s}$. Our analysis is based on images produced by the STSDAS multidrizzle package (Koekemoer et al. 2002), and the final images have a pixel scale of 0 '.05 $\mathrm{pixel}^{-1}$. For galaxies with half-light radii of 0 '.25, $0^{\prime \prime} .50$, and $1 . \prime 00$, the completeness is $50 \%$ at $\mathrm{F} 814 \mathrm{~W} \simeq 26.0,24.7$, and 24.5 , respectively.

\subsubsection{Redshifts}

For the COSMOS survey we provide 10,236 spectroscopic redshifts, of which 8472 are reasonably secure redshifts (confidence class 3.x, 4.x, 1.5, 2.4, 2.5, 9.3, 9.5, 13.x, 14.x, 23.x, and 24.x) from the zCOSMOS redshift survey (Lilly et al. 2009). The primary zCOSMOS targets were selected for spectroscopy from the "total" F814W magnitudes and were required to be in the magnitude range $15.0<\mathrm{F} 814 \mathrm{~W}<22.5$. The quality metrics used for the zCOSMOS survey are described in depth in Table 1 of Lilly et al. (2009). It is worth noting that only $\sim 5.0 \%$ of the reasonably secure redshifts are at $z \geqslant 1.0$; the majority of the spectroscopic redshifts are in the range of $0.2<z<1.0$. Approximately $88 \%$ of the galaxies observed in zCOSMOS have a spectroscopic redshift that is secure at the $99 \%$ level.

We provide 251,971 photometric redshifts from Ilbert et al. (2009). These highly accurate photometric redshifts are based on 30-band photometry, spanning the wavelength range of UV to mid-IR. Using a sample of 4148 galaxies from the zCOSMOS-bright survey, Ilbert et al. (2009) recover a catastrophic failure rate $\eta=0.7 \%$ and redshift accuracy of $\sigma_{|\Delta z| /(1+z \text { spec })}=0.007$ for $i^{+}<22.5$. Due to the magnitude limits probed by the zCOSMOS-bright survey, photometric redshift reliabilities for fainter magnitudes, $i^{+}>22.5$, where $i^{+}$refers to the Subaru photometric system, were tested using 209 galaxies from the zCOSMOS-faint survey and 317 galaxies from the MIPS spectroscopic sample (Kartaltepe et al. 2010). At high redshift $1.5<z<3.0$, Ilbert et al. (2009) recover a catastrophic failure rate $\eta=20.4 \%$ with a redshift accuracy $\sigma_{|\Delta z| /\left(1+z_{\text {spec }}\right)}=0.053$ with a median magnitude $i_{\text {median }}^{+}=24.0$. For $22.5<i^{+}<24.0$ they measure a redshift accuracy of $\sigma_{|\Delta z| /\left(1+z_{\text {sspec }}\right)}=0.011$. These results are summarized in Table 3 of Ilbert et al. (2009).

\subsection{The GEMS Survey}

\subsubsection{Imaging}

GEMS is an $800 \operatorname{arcmin}^{2}$ survey using the HST ACS instrument in two bands (V606W and F850LP; Rix et al. 2004, Caldwell et al. 2008). The field was chosen due to the rich set of observations at complementary wavelengths. GEMS is centered on the Extended Chandra Deep Field South $\left(\alpha=03^{\mathrm{h}} 32^{\mathrm{m}}\right.$, $\delta=-27^{\circ} 48^{\prime}$ ). The central $\sim 25 \%$ of the E-CDFS field has deep HST ACS imaging from the GOODS survey. The HST ACS imaging in the GEMS field is composed of 63 pointings using both the F606W and F850LP filters, with exposure times of 2160 and $2286 \mathrm{~s}$ per pointing, respectively. Our analysis is based on images produced by the STSDAS multidrizzle package. The final images have a pixel scale of $00^{\prime} .03$ pixel $^{-1}$. For an extended object the $5 \sigma$ limiting magnitudes are $\mathrm{F} 606 \mathrm{~W}=25.7(\mathrm{AB})$ and $\mathrm{F} 850 \mathrm{LP}=24.2(\mathrm{AB})$.

\subsubsection{Redshifts}

For the GEMS + GOODS-S surveys we provide spectroscopic redshifts from various sources (Table 2) and provide a total of 6955 spectroscopic redshifts, with 5756 high-quality redshifts $\left(z_{q} \geqslant 3\right)$. The quality of the redshifts ranges from 1 for the lowest quality to 4 for the highest quality redshifts. Refer to the catalog parameter Z_ORIGIN for the origin of the spectroscopic redshift (see Table 2 ).

We provide 44,239 photometric redshifts from the COMBO17 survey (Wolf et al. 2008). Using a high-quality subset of spectroscopic redshifts from Le Fèvre et al. (2004), they find the $\Delta z /\left(1+z_{\mathrm{s}}\right)$ deviations to have an $\mathrm{rms} \sim 0.008$ at $R<21$, 
Table 2

GEMS + GOODS-S Spectroscopic References

\begin{tabular}{ll}
\hline \hline z_origin & \\
\hline VLT_2008 & Vanzella et al. (2008) \\
VLT_IMAG & Ravikumar et al. (2007) \\
VLT_LBGs & Vanzella et al. (2009) \\
VIMOS_08_MR/LR & Popesso et al. (2009) \\
GRISM_HUDF & Hathi et al. (2008) and Rhoads et al. (2009) \\
ePEARS_HUDF & Straughn et al. (2008) \\
ePEARS_CDFS & Straughn et al. (2009) \\
GRAPES_HUDF & Hathi et al. (2009) and Pasquali et al. (2006) \\
K20 & Mignoli et al. (2005) \\
CXO-CDFS & Szokoly et al. (2004) \\
VVDS & Le Fèvre et al. (2004) \\
LCIRS & Doherty et al. (2005) \\
FW_5 & Norman et al. (2002) \\
FW_6 & Croom et al. (2001) \\
FW_7 & van der Wel et al. (2005) \\
FW_8 & Cristiani et al. (2000) \\
FW_9 & Strolger et al. (2004) \\
FW_10 & Daddi et al. (2004) \\
FW_13 & Wuyts et al. (2009) \\
FW_14 & Kriek et al. (2008) \\
FW_15 & Roche et al. (2006) \\
FW_16 & Wuyts et al. (2008) \\
ACES & Cooper et al. (2011b) \\
\hline
\end{tabular}

increasing to 0.02 at $R<23$ and 0.035 for $23.0<R<24.0$. Note, however, that not much is known about the photometric redshift accuracy for normal galaxies at $z>1.2$. Refer to Wolf et al. (2004) and Wolf et al. (2008) for a full description of these data.

\section{REDSHIFT COMPLETENESS AND RELIABILITY}

All extra-galactic surveys are fundamentally limited by the completeness in their spectroscopic and photometric redshifts. Referring to Table 3, we can see that the redshifts for each survey are dominated by the photometric redshifts and these will dominate the completeness of the redshift survey. Throughout this particular analysis we concentrate on the photometric redshift samples, focusing on the highest reliable photometric redshifts provided by each survey. In Figure 1, we plot the histograms of the photometric redshift errors provided by each survey. The AEGIS, GEMS, and GOODS-S are $1 \sigma$ and COSMOS is $3 \sigma$. We observe a peculiar bi-modal distribution for the GEMS and GOODS-S distribution. The photometric redshift errors for GOODS-N are larger than for the other surveys and users should exercise caution when using this sample. To select reliable photometric redshifts from AEGIS, GEMS, GOODS-S, and COSMOS, we require photoz_err $\leqslant 0.15 \times(1+$ photoz $)$.

Another reliability test that can be done is to compare high-quality spectroscopic redshifts to their photometric counterparts. We select all spectroscopic redshifts with $z_{q} \geqslant 3$, while the COSMOS high-quality redshifts are described in Section 2.3.2. In Figure 2, we plot the high-quality spectroscopic redshift versus the photometric redshift. We observe EGS and COSMOS to have highly consistent results, while GEMS and GOODS-S seem to have larger uncertainties at $z>1.0$. The GOODS-N sample appears to have the largest dispersions, and users should exercise caution when using this sample.

Having an unbiased estimation of the redshift completeness requires reliably removing compact sources and low surface brightness (LSB) galaxies from the sample. This is performed by utilizing the method described in Section 5.5. Using a

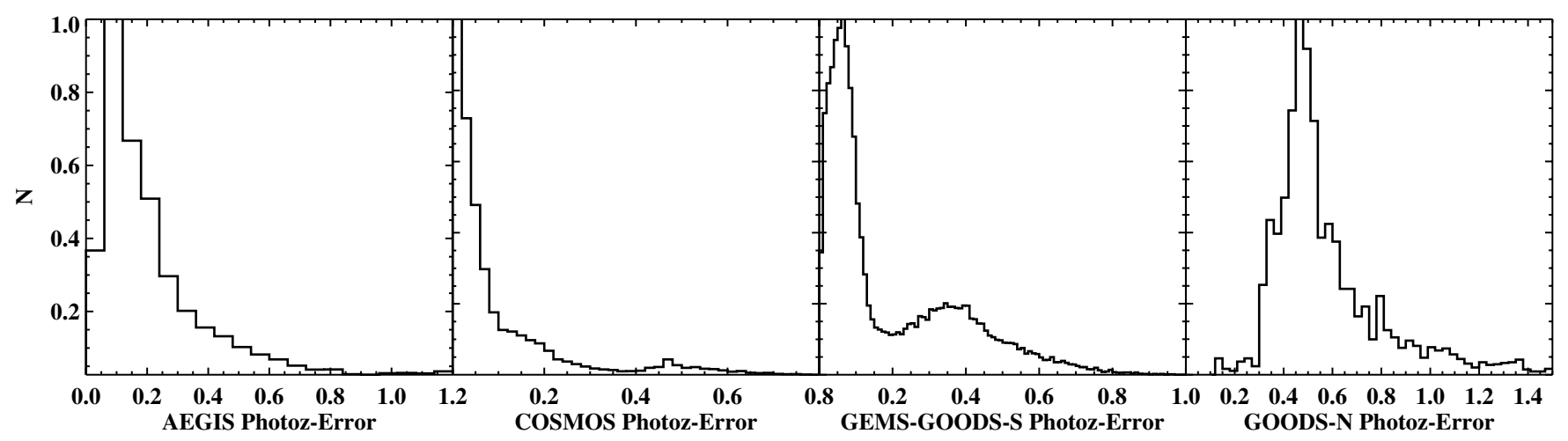

Figure 1. Histograms of the photometric redshift errors in the ACS-GC surveys.

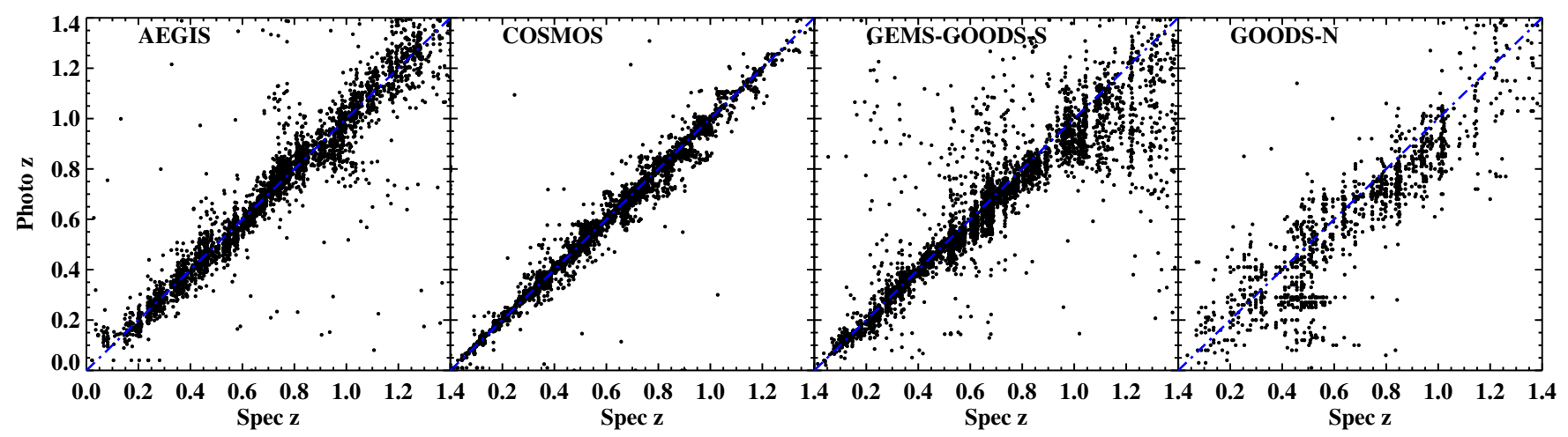

Figure 2. Spectroscopic redshift vs. photometric redshift in the ACS-GC surveys.

(A color version of this figure is available in the online journal.) 


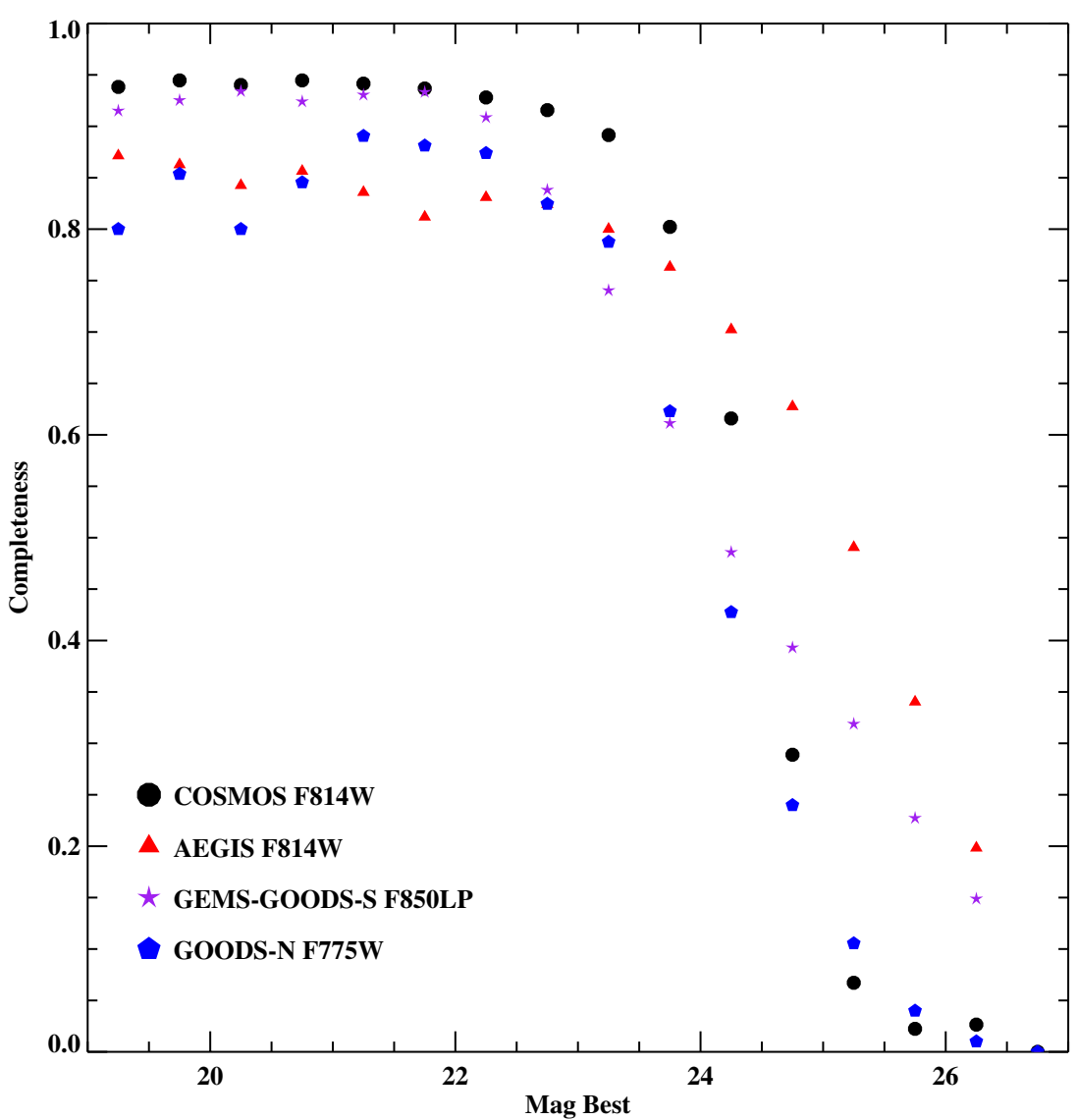

Figure 3. Mag Best vs. completeness for all surveys in the ACS-GC. The selection of the photometric redshifts is described in Section 3.

(A color version of this figure is available in the online journal.)

sample of normal extended galaxies with reliable photometric redshift estimates, we can estimate the redshift completeness as a function of magnitude in the following manner. The completeness for a given magnitude bin ( $\Delta$ Mag 0.5$)$ is given by

$$
C(\operatorname{mag})=\frac{N(z)}{N(\text { total })} .
$$

In Figure 3, we plot the photometric redshift completeness as a function of magnitude for all surveys. For AEGIS and COSMOS we plot F814W, for GEMS and GOODS-S we plot F850LP, and for GOODS-N we plot F775W. For COSMOS we can see that the sample is highly complete to F814W $<23.5$ and dropping to $75 \%$ at $\mathrm{F} 814 \mathrm{~W}=24.0$. AEGIS is highly complete to $\mathrm{F} 814 \mathrm{~W}<23.0$ and drops to $70 \%$ at F814 $=24.0$. GEMS and GOODS-S are a bit shallower than AEGIS and COSMOS, being $75 \%$ complete at F850LP $=23.5$. For GOODS-N we did not apply any reliability criteria and compute the completeness with all available measurements. We can see that GOODS-N is $75 \%$ complete at $\mathrm{F} 775 \mathrm{~W}=23.5$.

\section{GALAXY PHOTOMETRY AND QUANTITATIVE MORPHOLOGY}

In order to combine and analyze this extremely large imaging data set, we adopted an automated fitting method called Galapagos. Galapagos was written in the IDL language to analyze large ACS imaging data sets through the GALFIT code (Häußler et al. 2011). The code was tested and compared to the Galaxy Image 2D (GIM2D) (Simard 1998) code by Häußler et al. (2007) using the F850LP GEMS ACS imaging. They conclude that GALFIT is more robust in crowded fields since it does simultaneous fitting of nearby galaxies, a capability not available with GIM2D. GALFIT and GIM2D use different convergence methods, and Häußler et al. (2007) found that GALFIT operated faster than GIM2D in analyzing these large imaging surveys. Galapagos is structured into four program blocks: SExtraction, postage stamp cutting, sky estimation and GALFIT, and catalog creation. The code is controlled mainly through a setup script and a file location list. Refer to Häußler et al. (2007) for a detailed description of GALAPAGOS. We next give a brief description of our SExTRACTOR and GaLFIT setup.

\subsection{SEXTRACTOR}

We use SExtractor (Bertin \& Arnouts 1996) to create the photometric catalogs used as initial inputs given to GALFIT. SEXTRACTOR detects, deblends, measures, and classifies objects, giving estimates of magnitude, size, axis ratio $(b / a)$, position angles, and a star-galaxy classification. The GEMS team found that no single SEXTRACTOR setup satisfactorily detected and deblended both bright, well-resolved galaxies and faint galaxies near the detection limit. Accordingly, the best setup found by GEMS was to run SExTRACTOR twice: once to detect bright objects without splitting them up (what is called the "cold" mode) and once to detect faint objects ("hot" mode). The two modes are then combined to give one single catalog containing all objects. The procedure is described in detail in Rix et al. (2004) and Caldwell et al. (2008). We use the final combined catalog to provide GALFIT with initial input parameters. 
Table 3

Catalog Statistics

\begin{tabular}{lcccccc}
\hline \hline Survey & Objno & Filter & Ntot & specz & specz $\left(z_{q} \geqslant 3\right)$ & photoz \\
\hline AEGIS & 1xxxxxxx & F606W & 65,301 & 5,691 & 4,244 & 41,982 \\
& & F814W & 55,808 & 5,691 & 4,244 & 37,294 \\
& & F606W+F814W & 50,967 & 5,691 & 4,244 & 35,480 \\
\hline COSMOS & $2 x x x x x x$ & F814W & 304,688 & 10,236 & $8,472^{\mathrm{a}}$ & 251,971 \\
\hline \multirow{2}{*}{ GOODS-N } & 5xxxxxxx & F606W & 23,071 & 2,793 & 1,332 & 6,051 \\
& & F775W & 17,592 & 2,832 & 1,343 & 6,128 \\
& & F606W+F775W & 16,438 & 2,771 & 1,328 & 5,901 \\
\hline GEMS-GOODS-S & 9xxxxxxx & F606W & 63,321 & 6,792 & 5,639 & 42,942 \\
& & F850LP & 54,613 & 6,781 & 5,694 & 37,613 \\
& & F606W+F850LP & 47,488 & 6,618 & 5,577 & 36,316 \\
\hline
\end{tabular}

Note. ${ }^{\text {a }}$ See Section 2.3.2 for a description of this sample.

Table 4

Object Numbers

\begin{tabular}{lll}
\hline \hline Survey & \multicolumn{1}{c}{ Objno } & \multicolumn{1}{c}{ Description } \\
\hline AEGIS & $100 \times x x x x$ & F814W and F606W detection in ACS-GC but not DEEP2 \\
& $101 \times x x x x$ & F814W detection only in ACS-GC but not DEEP2 \\
& $102 \times x x x x$ & F606W detection only in ACS-GC but not DEEP2 \\
$1(1 / 2 / 3 / 4) 0 x x x x$ & F814W and F606W Detection in ACS-GC and DEEP2 \\
\hline COSMOS & $20 \times x x x x$ & F814W detection \\
\hline GOODS-N & $500 \times x x x x$ & F775W and F606W detection \\
& $501 \times x x x x$ & F775W detection only \\
& $502 \times x x x x$ & F606W detection only \\
\hline \multirow{2}{*}{ GEMS + GOODS-S } & $900 \times x x x x$ & F606W and F850LP detection \\
& $901 \times x x x x$ & F850LP detection only \\
& $902 \times x x x x$ & F606W detection only \\
\hline
\end{tabular}

\subsection{GALFIT}

GALFIT is designed to measure structural parameters from galaxy images. We model each source in the catalog with a single Sérsic profile as well as a model for the sky (which we keep fixed during the fit). The Sérsic profile (1968) is defined as

$$
\Sigma(r)=\Sigma_{e} e^{-k\left[\left(r / r_{e}\right)^{1 / n}-1\right]},
$$

where $r_{e}$ is the effective radius of the galaxy, $\Sigma_{e}$ is the surface brightness at $r_{e}, n$ is the Sérsic index, and $k$ is coupled to $n$ such that half of the total flux is always within $r_{e}$. Before evaluating its fit to the data, GALFIT convolves the two-dimensional image with a point-spread function (PSF), derived empirically from a high signal-to-noise star, with a single PSF used for each band and survey. GALFIT then uses a Levenberg-Marquardt algorithm for $\chi_{v}^{2}$ minimization. The Sérsic profile has seven free parameters: $x$-center, $y$-center, position angle, Sérsic index, half-light radius, axis ratio, and magnitude. GALFIT requires a setup script, which is created by GALAPAGOS, which has initial guesses for many of the parameters. In particular, using SExtractor parameters, starting magnitudes were given by MAG_BEST, and sizes were derived from the FLUX_RADIUS using the formula $r_{e}=0.162 R_{\text {flux }}^{1.87}$, where $R_{\text {flux }}$ is FLUX_RADIUS. This formula was determined empirically using simulations. The axis ratio $b / a$ and the position angle were derived by taking the SExtractor parameters ELLIPTICITY and THETA_IMAGE, respectively. Furthermore, the position of each object within its postage stamp was required as an input parameter for GALFIT, which was directly given by the process of cutting the postage stamps (the object is centered within its postage stamp). See Häußler et al. (2007) for a more detailed description of this process. Our initial input for the Sérsic index was 2.5. GALFIT produces a summary of the fit parameters and a FITS image block that includes the original image, the model image, and the residual image (original-model).

\section{THE CATALOG}

For each ACS survey we combined the SExTRACTOR, GALFIT, and redshift catalogs to produce a single combined catalog. We then combined all of the surveys to produce the single, uniformly constructed ACS-GC catalog. This catalog has 97 parameters, in order to provide a comprehensive list of galaxy properties. We use an NGC-style numbering scheme; refer to Section 5.1 and Table 4. Table 5 presents a description of the parameters. The naming convention is similar to the DEEP 2 redshift survey. We also unite the photometry and structural measurements for the different surveys in a consistent manner by appending _HI and_LOW to parameters that were measured in the individual ACS filters, where _LOW refers to the F606W filter while_HI is F850LP for GEMS and GOODS-S, F775W for GOODS-N, and F814W for COSMOS and AEGIS. The catalog parameter IMAGING gives the origin of the ACS imaging used to measure the parameters of interest and is useful for separating GEMS and GOODS-S.

Table 3 gives basic catalog statistics, e.g., object numbers, filters, total number of sources identified in each filter (Ntot), 

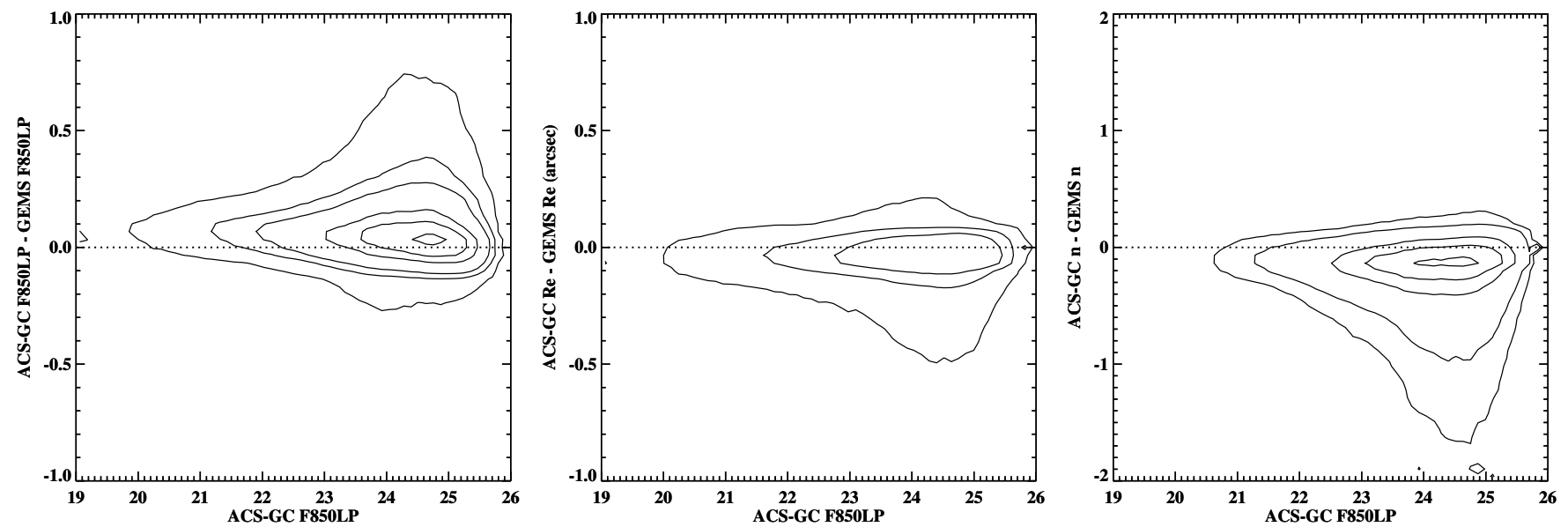

Figure 4. Left: $\Delta$ Mag GaLFIT vs. ACS-GC F850LP. Center: $\Delta r_{e}$ vs. ACS-GC F850LP. Right: $\Delta n$ vs. ACS-GC F850LP.

total number of spectroscopic redshifts, total number of highquality spectroscopic redshifts $\left(z_{q} \geqslant 3\right)$, and total number of photometric redshifts in each filter.

\subsection{Object Identification}

Our object identification scheme has been adopted from the DEEP2 survey, which uses an 8 digit number to identify each source in the catalog. The convention is motivated by the fact that each input survey uses its own naming convention. Combining these surveys into one homogeneous data set required creating a single, uniform naming convention across all surveys. Table 4 gives a description of the object numbers and naming convention for the individual surveys.

We also supply the "SURVEY_ID" parameter in the ACS-GC catalog, which is the ID number used by the original survey. This allows users to easily and rapidly match the ACS-GC catalog, rather than having to cross-correlate catalogs using positions. This parameter is given, where available, for the AEGIS, COSMOS, and GOODS-N surveys. We do not provide this for GEMS and GOODS-S due to the naming convention used by these teams, which was using the source position, R.A., and decl. as the source ID.

To improve computational efficiency, some of the fields were divided into tiles with a small overlap between them, to ensure no objects were lost. Because of this, some objects appear more than once when merging catalogs of sources in the ACSGC. These duplications were removed by coordinate matching and visual inspection. Nevertheless, some repeated objects may still exist in the final catalogs, but the number should be very small and will be completely dominated by objects close to the faint detection limit.

\subsection{Flags}

We use a very simple method to distinguish whether a source has a good fit $(F L A G=0)$ or an unreliable fit $(F L A G=1)$. We use the GALFIT uncertainties for both the half-light radius and the Sérsic index $n$, and we use CLASS_STAR to separate extended sources from compact sources. Our good fits $(\mathrm{FLAG}=0)$ require $\sigma(n) \leqslant 0.15 * n, \sigma\left(r_{e}\right) \leqslant 0.15 * r_{e}$, and CLASS_STAR $\leqslant 0.8$. The additional requirement given by CLASS_STAR assigns unreliable results for both stellar-like and compact objects. As the source size becomes comparable to the PSF size, the results become increasingly unreliable. Since this is a very simple cut using few uncertainty parameters, the users of this data set are advised to use as many uncertainty parameters $\left(\chi_{\nu}^{2}\right.$, surface brightness, magnitude-limited samples, etc.) to define high-quality samples for their investigations.

\subsection{Reliability and Measurement Errors}

Structural parameter errors quoted in the ACS-GC come directly from the GALFIT fitting results. It is worth noting that Häußler et al. (2007) found that GALFIT substantially underestimated the true fit uncertainties, indicating that the dominant contribution to the fitting uncertainties is not shot and read noise, but instead contamination from neighbors, structure in the sky, correlated pixels, profile mismatch, etc. They also find that the reliability of the fitting results was dependent on the galaxy type measured. For galaxies with exponential profiles $(n=1.00)$ and brighter than the sky's surface brightness, they found no significant mean offset between the input and recovered parameters. For galaxies exhibiting a de Vaucouleurs profile $(n=4.00)$, they find that GALFIT recovers parameters that are significantly less accurate than the $n=1.00$ galaxies. This behavior is attributed to two factors. First, spheroidal profiles are in principle harder to fit due to the importance of the outskirts of the light profile, thus requiring a careful and accurate measurement of the sky background to be used in order to return a reliable fit. Second, due to the large amount of light in the faint wings of the galaxies, neighboring objects have a much bigger influence on the fit of the galaxy of interest.

As a sanity check on the structural parameters of the ACS-GC, we compare the results from the ACS-GC GEMS F850LP imaging to the results obtained in Häußler et al. (2007). Figure 4 shows the comparison between these two analyses. Left plot gives $\Delta \mathrm{F} 850 \mathrm{LP}$ versus F850LP, center plot gives $\Delta r_{e}$ versus F850LP, and right plot gives $\Delta n$ versus F850LP. As expected, we observe a clear systematic trend in the differences of the recovered parameters as a function of magnitude. The recovered parameters are highly consistent to F850LP $\leqslant 24.0$. Sources with F850LP $\geqslant 24.0$ show larger systematic differences, especially the magnitudes and Sérsic index measurements. These results show that for galaxies above the sky's surface brightness the recovered parameters are generally reliable, but for fainter galaxies users should apply caution when using the derived parameters. 


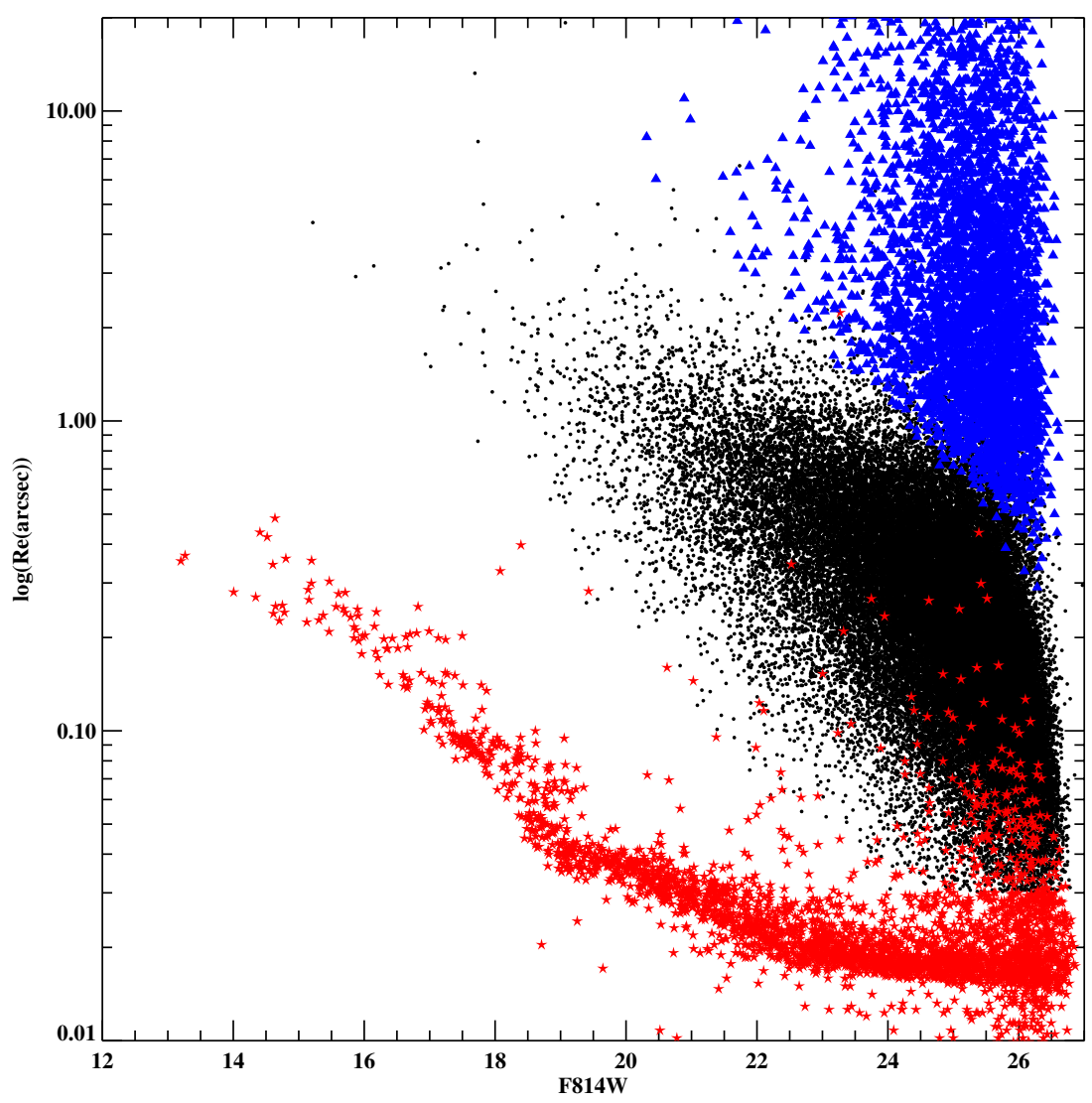

Figure 5. F814W vs. $\log r_{e}^{\prime \prime}$ for all galaxies in the AEGIS survey with detections in the F814W filter. Red stars are compact sources, black dots are extended sources, and blue triangles are LSB galaxies.

(A color version of this figure is available in the online journal.)

\subsection{Auxiliary Parameters}

In addition to parameters measured with the ACS images, we provide a few additional useful parameters. We include the CFHTLS $(u, g, r, i, z)$ photometry (COSMOS and AEGIS) (Gwyn 2008). We also provide BRI magnitudes for both COSMOS (Capak et al. 2007) and AEGIS (Davis et al. 2007). The parameter Ntot, which was derived during the catalog creation process, gives the number of sources that were simultaneously fit with GALFIT while fitting the primary source. This could be used to investigate line-of-sight overdensities in the ACS imaging. We also supply the surface brightness, defined as

$$
\mu=\operatorname{mag}+2.5 \cdot\left(\log \left(2 \cdot b / a \cdot \pi \cdot\left(r_{e}\right)^{2}\right),\right.
$$

where mag is given by Mag Best, $b / a$ is the axis ratio, and $r_{e}$ is given in arcseconds. These parameters can be useful in the investigation of detailed galaxy properties and selecting complete and reliable samples; see Section 5.5.

\subsection{Compact and Extended Sources in the ACS-GC}

It has been known that the CLASS_STAR parameter returned by SEXTRACTOR is problematic in reliably distinguishing compact stellar-like sources and extended sources in imaging surveys. By defining compact objects as those having $\mu \leqslant 18$ or $\left(\mu \geqslant 18\right.$ and $\left.r_{e} \leqslant 00^{\prime} 03\right)$, we easily circumvent this issue. We demonstrate the reliability of this definition in Figure 5, where we plot all sources in the AEGIS survey having an F814W detection, with the $x$-axis representing the F814W magnitude and the $y$-axis the half-light radius $r_{e}$ given in arcseconds. Red stars represent compact sources (by our definition), and black circles represent extended sources. There is, however, another class of galaxies that have been notorious for producing unreliable results: these are the LSB galaxies and tend to populate the top right-hand corner of the magnitude-size diagram. These can easily be removed by requiring the extended galaxies to have $\mu<26.0$. The extended galaxies with $\mu>26.0$ are represented by the blue triangles in Figure 5 and are considered to be LSB galaxies. We can see that these definitions do an excellent job in distinguishing between these three populations. Similar cuts can be applied to all the ACS-GC surveys to separate compact sources from extended sources and LSB galaxies.

\subsection{GALFIT Residual Maps, Color Images, and The Galaxy Atlas}

We provide high-resolution ACS pseudocolor images for the GEMS, AEGIS, and GOODS surveys, from which twoband imaging was available. These RGB images were made using the F814W and F606W images for the AEGIS data, the F850LP and F606W images for GEMS + GOODS-S, and the F775W and F606W images for GOODS-N. For example, the AEGIS color images were made using the following convention: the red channel was assigned to the F814W image, the blue channel was assigned to the F606W image, and the green channel was assigned to $(\mathrm{F} 814 \mathrm{~W}+\mathrm{F} 606 \mathrm{~W}) / 2$. These individual images were then converted into color images using the IDL routine djs_rgb_make.pro (D. Schlegel 2007, private communication). The COSMOS survey only has a single ACS band $(\mathrm{F} 814 \mathrm{~W})$, thus making it impossible to derive ACS 


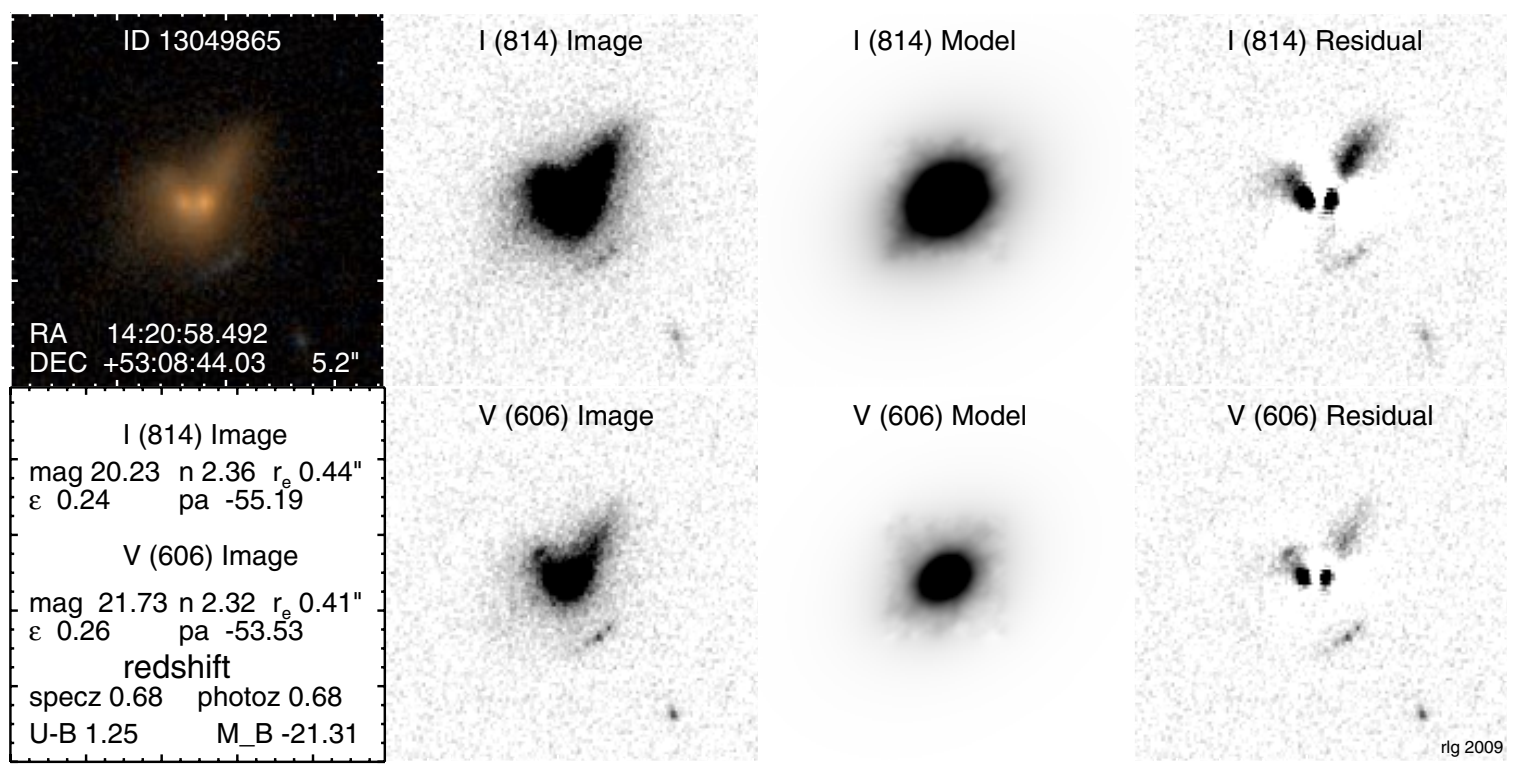

Figure 6. Example ACS-GC atlas image, as described in Section 5.6.

(A color version of this figure is available in the online journal.)

high-resolution color images. However, pseudocolor images in the COSMOS field were constructed by P. Capak using the ACS F814W data as an illumination map and the Subaru $B_{J}, r^{+}$, and $i^{+}$images as a color map. To achieve this, each Subaru image was divided by the average of the three Subaru images and then multiplied by the ACS F814W image. This preserves the flux ratio between images while replacing the overall illumination pattern with the F814W data. Each image was then divided by $\lambda^{2}$ to enhance the color difference between star-forming and passive galaxies. The processed $B_{J}, r^{+}$, and $i^{+}$images were then assigned to the blue, green, and red channels, respectively. The resulting images have the high spatial resolution of the ACS imaging but color gradients at ground-based resolution. For every source in the ACS-GC catalog we provide a high-resolution color image as well as the original ACS FITS images used to make the color images. For COSMOS we also provide the Subaru images used to make the color images.

For every source fitted by GALFIT, GALFIT returns a FITS image block that contains four extensions. Extension $=0$ is blank, extension $=1$ is the original ACS image, extension $=2$ is the GALFIT model image, and extension $=3$ is the GALFIT residual image (model-original). These residual images are useful for many applications. For example, they can be used to identify rare classes of galaxies, such as gravitational lenses, ring galaxies, dual AGNs (Comerford et al. 2009), and mergers. The residual maps also allow a visual confirmation of the quality of the fit. For every source in the ACS-GC catalog we provide this GALFIT image block. For the GEMS, AEGIS, and GOODS survey this is generally two files, one for each band. The COSMOS single-band imaging produces only one of these files.

We combine these secondary imaging data and key structural parameters into a single file for each source, the atlas image. Figure 6, which shows the atlas image of ACS-GC 13049865 (AEGIS) as an example, provides the ACS color image (top left) and the GALFIT image blocks with the redder band in the top row and the bluer band, when available, as the lower row. The color panel provides the object number, R.A., decl., and the field of view in arcseconds. The bottom left panel gives key parameters for the source, including the magnitude (Mag Best), the Sérsic index $(n)$, the half-light radius $\left(r_{e}\right)$, the ellipticity $(\epsilon)$, and the position angle (P.A.) for each band analyzed. We also give the spectroscopic and photometric redshifts (when available) and the $U-B$ rest-frame color and absolute $B$-band magnitude.

\section{SUMMARY}

In this work, we have measured photometric and structural parameters for roughly half a million galaxies contained within the largest HST ACS extragalactic imaging surveys obtained to date. These surveys have not been analyzed in a consistent manner previously. The unified analysis presented here opens the possibility for scientific investigations that rely on these multiple fields being analyzed in a consistent manner. We publicly release the ACS-GC catalog, which includes 97 parameters for 469,501 astronomical sources, as well as secondary science products such as color images, GALFIT images, atlas images, and FITS images (with WCS). Additional data products are expected in the near future from the Galaxy Zoo ${ }^{16}$ project, which plans to visually classify a large fraction of the ACS-GC color images. The ultimate goal of the ACS-GC galaxy morphology data set is to provide a statistically significant sample of galaxies that can be used to investigate detailed galaxy properties, as well as to understand how galaxy structures evolve over cosmic times.

We gratefully acknowledge the principal investigators responsible for the ACS imaging utilized by the ACS-GC. We also acknowledge the GEMS team for creating and sharing the GALAPAGOS code, without which none of this would have been possible. Work on this paper was carried out at the Jet Propulsion Laboratory and Infrared Processing and Analysis Center (IPAC), California Institute of Technology, under contract with NASA and WISE. J.M.C. is supported by an NSF Astronomy and Astrophysics Postdoctoral Fellowship under award

\footnotetext{
16 www.galaxyzoo.org
} 
Table 5

ACS-GC Catalog Parameter Description

\begin{tabular}{|c|c|c|}
\hline No. & Parameter & Description \\
\hline 1 & OBJNO & Unique object number \\
\hline 2 & SURVEY_ID & The unique survey ID, if available \\
\hline 3 & RA & Right ascenion J2000 in decimal degrees \\
\hline 4 & $\mathrm{DEC}$ & Declination J2000 in decimal degress \\
\hline 5 & NTOT_HI & Total number of objects simultaneously fitted \\
\hline 6 & NTOT_LOW & Total number of objects simultaneously fitted \\
\hline 7 & IMAGING & Imaging survey \\
\hline 8 & SPECZ & Spectroscopic redshift \\
\hline 9 & PHOTOZ & Photometric redshift \\
\hline 10 & PHOTOZ_CHI2 & Reduced chi 2 for photometric redshift \\
\hline 11 & PHOTOZ_ERR & For EGS $(1 \sigma)$ and COSMOS $(3 \sigma)$ \\
\hline 12 & ZQUALITY & The quality flag for spectroscopic redshift \\
\hline 13 & Z_ORIGIN & Origin of spectroscopic redshift \\
\hline 14 & $\mathrm{Z}$ & High-quality specz else use photoz \\
\hline 15 & MAGB & $B$-band apparent magnitude \\
\hline 16 & MAGB_ERR & Error is $B$-band magnitude \\
\hline 17 & MAGR & $R$-band apparent magnitude \\
\hline 18 & MAGR_ERR & Error in $R$-band magnitude \\
\hline 19 & MAGI & $I$-band apparent magnitude \\
\hline 20 & MAGI_ERR & Error in $I$-band magnitude \\
\hline 21 & CFHT_U & CFHTLS $u$ mag \\
\hline 22 & CFHT_U_ERR & CFHTLS $u$ mag error \\
\hline 23 & CFHT_G & CFHTLS $g$ mag \\
\hline 24 & CFHT_G_ERR & CFHTLS $g$ mag error \\
\hline 25 & CFHT_R & CFHTLS $r$ mag \\
\hline 26 & CFHT_R_ERR & CFHTLS $r$ mag error \\
\hline 27 & CFHT_I & CFHTLS I mag \\
\hline 28 & CFHT_I_ERR & CFHTLS I mag error \\
\hline 29 & CFHT_Z & CFHTLS $z$ mag \\
\hline 30 & CFHT_Z_ERR & CFHTLS $z$ mag error \\
\hline 31 & EBV & Extinction \\
\hline 32 & CLASS & Object classification, provided by DEEP2 survey and COMBO-17 survey \\
\hline 33 & MU_HI & Surface brightness \\
\hline 34 & MU_LOW & Surface brightness \\
\hline 35 & THETA_IMAGE_HI & Theta image (SEXTRACTOR) \\
\hline 36 & THETA_IMAGE_LOW & Theta image (SExtractor) \\
\hline 37 & THETA_WORLD_HI & Theta world (SEXTRACTOR) \\
\hline 38 & THETA_WORLD_LOW & Theta world (SEXTRACTOR) \\
\hline 39 & BA_HI & Axis ratio $b / a$ (SEXTRACTOR) \\
\hline 40 & BA_LOW & Axis ratio $b / a$ (SEXTRACTOR) \\
\hline 41 & KRON_RADIUS_HI & Kron radius (SEXTRACTOR) \\
\hline 42 & KRON_RADIUS_LOW & Kron radius (SEXTRACTOR) \\
\hline 43 & FWHM_HI & Full width at half-maximum (SEXTRACTOR) \\
\hline 44 & FWHM_LOW & Full width at half-maximum (SEXTRACTOR) \\
\hline 45 & A_IMAGE_HI & $A$ axis (SEXTRACTOR) \\
\hline 46 & A_IMAGE_LOW & $A$ axis (SEXTRACtor) \\
\hline 47 & B_IMAGE_HI & $B$ axis (SEXTRACTOR) \\
\hline 48 & B_IMAGE_LOW & $B$ axis (SExtractor) \\
\hline 49 & BACKGROUND_HI & Sky background (SEXTRACTOR) \\
\hline 50 & BACKGROUND_LOW & Sky background (SEXTRACTOR) \\
\hline 51 & FLUX_BEST_HI & Flux best (SEXTractor) \\
\hline 52 & FLUX_BEST_LOW & Flux best (SEXTractor) \\
\hline 53 & FLUXERR_BEST_HI & Error in flux best (SEXTRACTOR) \\
\hline 54 & FLUXERR_BEST_LOW & Error in flux best (SEXTRACTOR) \\
\hline 55 & MAG_BEST_HI & Mag best (SEXTRACTOR) \\
\hline 56 & MAG_BEST_LOW & Mag best (SEXTRACTOR) \\
\hline 57 & MAGERR_BEST_HI & Error in mag best (SEXTRACTOR) \\
\hline 58 & MAGERR_BEST_LOW & Error in mag best (SEXTRACTOR) \\
\hline 59 & FLUX_RADIUS_HI & Flux radius (SEXTRACTOR) \\
\hline 60 & FLUX_RADIUS_LOW & Flux radius (SEXTRACTOR) \\
\hline 61 & ISOAREA_IMAGE_HI & Iso area of object (SEXTRACTOR) \\
\hline 62 & ISOAREA_IMAGE_LOW & Iso area of object (SEXTRACTOR) \\
\hline 63 & SEX_FLAGS_HI & SEXTRACTOR flag \\
\hline 64 & SEX_FLAGS_LOW & SEXTRACTOR flag \\
\hline 65 & FLAG_GALFIT_HI & Flag GALFIT good $=0 \mathrm{bad}=1$ \\
\hline
\end{tabular}


Table 5

(Continued)

\begin{tabular}{|c|c|c|}
\hline No. & Parameter & Description \\
\hline 66 & FLAG_GALFIT_LOW & Flag GALFIT good $=0 \mathrm{bad}=1$ \\
\hline 67 & CHI2NU_HI & GALFIT reduced chi2 \\
\hline 68 & CHI2NU_LOW & GALFIT reduced chi2 \\
\hline 69 & CLASS_STAR_HI & Class star (SEXTRACTOR) \\
\hline 70 & CLASS_STAR_LOW & Class star (SEXTRACTOR) \\
\hline 71 & X_GALFIT_HI & $\mathrm{X}$ center for GALFIT residual image \\
\hline 72 & X_GALFIT_LOW & $\mathrm{X}$ center for GALFIT residual image \\
\hline 73 & Y_GALFIT_HI & Y center for GALFIT residual image \\
\hline 74 & Y_GALFIT_LOW & Y center for GALFIT residual image \\
\hline 75 & MAG_GALFIT_HI & Mag (GALFIT) \\
\hline 76 & MAG_GALFIT_LOW & Mag (GALFIT) \\
\hline 77 & RE_GALFIT_HI & Effective half-light radius (GALFIT) \\
\hline 78 & RE_GALFIT_LOW & Effective half-light radius (GALFIT) \\
\hline 79 & N_GALFIT_HI & Sérsic index $[n<1.5$ (Late type) $n>2.5$ (Early type) (GALFIT) \\
\hline 80 & N_GALFIT_LOW & Sérsic index $[n<1.5$ (Late type) $n>2.5$ (Early type) (GaLFIT) \\
\hline 81 & BA_GALFIT_HI & Axis ratio (GALFIT) \\
\hline 82 & BA_GALFIT_LOW & Axis ratio (GALFIT) \\
\hline 83 & PA_GALFIT_HI & Position angle (GALFIT) \\
\hline 84 & PA_GALFIT_LOW & Position angle (GALFIT) \\
\hline 85 & SKY_GALFIT_HI & Sky background measured by the GALAPAGOS code \\
\hline 86 & SKY_GALFIT_LOW & Sky background measured by the GALAPAGOS code \\
\hline 87 & MAGERR_GALFIT_HI & Error in mag (GALFIT) \\
\hline 88 & MAGERR_GALFIT_LOW & Error in mag (GALFIT) \\
\hline 89 & REERR_GALFIT_HI & Error in half-light radius (GALFIT) \\
\hline 90 & REERR_GALFIT_LOW & Error in half-light radius (GALFIT) \\
\hline 91 & NERR_GALFIT_HI & Error in Sérsic index (GALFIT) \\
\hline 92 & NERR_GALFIT_LOW & Error in Sérsic index (GALFIT) \\
\hline 93 & BAERR_GALFIT_HI & Error in axis ratio (GALFIT) \\
\hline 94 & BAERR_GALFIT_LOW & Error in axis ratio (GALFIT) \\
\hline 95 & PAERR_GALFIT_HI & Error in position angle (GALFIT) \\
\hline 96 & PAERR_GALFIT_LOW & Error in position angle (GALFIT) \\
\hline 97 & VIS_MORPH & Visual morphology classification (currently not available) \\
\hline
\end{tabular}

(A supplementary tar.gz file containing the complete data of this table is available in the online journal.)

AST-1102525. We thank the anonymous referee for his/her useful suggestions, which have improved this manuscript.

This work is based on (GO-10134, GO-09822, GO-09425.01, GO-09583.01, GO-9500) program observations with the NASA/ESA Hubble Space Telescope, obtained at the Space Telescope Science Institute, which is operated by the Association of Universities for Research in Astronomy, Inc., under NASA contract NAS 5-26555. Work on this paper is based on observations obtained with MegaPrime/MegaCam, a joint project of CFHT and CEA/DAPNIA, at the Canada-FranceHawaii Telescope (CFHT), which is operated by the National Research Council (NRC) of Canada, the Institut National des Science de l'Univers of the Centre National de la Recherche Scientifique (CNRS) of France, and the University of Hawaii. This work is based in part on data products produced at TERAPIX and the Canadian Astronomy Data Centre as part of the Canada-France-Hawaii Telescope Legacy Survey, a collaborative project of NRC and CNRS. Funding for the DEEP2 Galaxy Redshift Survey has been provided in part by NSF grant AST00-71048 and NASA LTSA grant NNG04GC89G. This work is also based on $z$ COSMOS observations carried out using the Very Large Telescope at the ESO Paranal Observatory under Program ID: LP175.A-0839. Some of the data presented herein were obtained at the W. M. Keck Observatory, which is operated as a scientific partnership among the California Institute of Technology, the University of California, and the National Aeronautics and Space Administration.

\section{REFERENCES}

Barger, A. J., Cowie, L. L., \& Wang, W. 2008, ApJ, 689, 687

Bertin, E., \& Arnouts, S. 1996, A\&AS, 117, 393

Bundy, K., Fukugita, M., Ellis, R. S., et al. 2009, ApJ, 697, 1369

Caldwell, J. A. R., McIntosh, D. H., Rix, H.-W., et al. 2008, ApJS, 174, 136

Capak, P., Aussel, H., Ajiki, M., et al. 2007, ApJS, 172, 99

Clampin, M., Ford, H., Illingworth, G., et al. 2002, BAAS, 34, 745

Comerford, J. M., Griffith, R. L., Gerke, B. F., et al. 2009, ApJ, 702, L82

Cooper, M. C., Aird, J. A., Coil, A. L., et al. 2011a, ApJS, 193, 14

Cooper, M. C., Griffith, R. L., Newman, J. A., et al. 2012, MNRAS, 419, 3018

Cooper, M. C., Yan, R., Dickinson, M., et al. 2011b, arXiv:1112.0312

Coupon, J., Ilbert, O., Kilbinger, M., et al. 2009, A\&A, 500, 981

Cowie, L. L., Barger, A. J., Hu, E. M., Capak, P., \& Songaila, A. 2004, AJ, 127, 3137

Cristiani, S., Appenzeller, I., Arnouts, S., et al. 2000, A\&A, 359, 489

Croom, S. M., Warren, S. J., \& Glazebrook, K. 2001, MNRAS, 328, 150

Daddi, E., Cimatti, A., Renzini, A., et al. 2004, ApJ, 617, 746

Davis, M., Faber, S. M., Newman, J., et al. 2003, Proc. SPIE, 4834, 161

Davis, M., Guhathakurta, P., Konidaris, N. P., et al. 2007, ApJ, 660, L1

Dickinson, M., Giavalisco, M., \& GOODS Team, 2003, in The Mass of Galaxies at Low and High Redshift, ed. R. Bender \& A. Renzini (Berlin: Springer), 324

Doherty, M., Bunker, A. J., Ellis, R. S., \& McCarthy, P. J. 2005, MNRAS, 361, 525

Georgakakis, A., Coil, A. L., Laird, E. S., et al. 2009, MNRAS, 397, 623

Giavalisco, M., Ferguson, H. C., Koekemoer, A. M., et al. 2004, ApJ, 600, L93

Griffith, R. L., \& Stern, D. 2010, AJ, 140, 533

Gwyn, S. D. J. 2008, PASP, 120, 212

Hathi, N. P., Ferreras, I., Pasquali, A., et al. 2009, ApJ, 690, 1866

Hathi, N. P., Malhotra, S., \& Rhoads, J. E. 2008, ApJ, 673, 686

Häußler, B., Barden, M., Bamford, S. P., \& Rojas, A. 2011, in ASP Conf. Ser. 442, Astronomical Data Analysis Software and Systems XX, ed. I. N. Evans, A. Accomazzi, D. J. Mink, \& A. H. Rots (San Francisco, CA: ASP), 155 
Häußler, B., McIntosh, D. H., Barden, M., et al. 2007, ApJS, 172, 615 Holden, B., van der Wel, A., Rix, H.-W., \& Franx, M. 2012, ApJ, 749, 96 Hubble, E. P. 1926, ApJ, 64, 321

Ilbert, O., Capak, P., Salvato, M., et al. 2009, ApJ, 690, 1236

Kartaltepe, J. S., Sanders, D. B., Le Floc'h, E., et al. 2010, ApJ, 709, 572

Koekemoer, A. M., Aussel, H., Calzetti, D., et al. 2007, ApJS, 172, 196

Koekemoer, A. M., Fruchter, A. S., Hook, R. N., \& Hack, W. 2002, in The 2002 HST Calibration Workshop: Hubble after the Installation of the ACS and the NICMOS Cooling System, ed. S. Arribas, A. Koekemoer, \& B. Whitmore, 337

Kriek, M., van Dokkum, P. G., Franx, M., et al. 2008, ApJ, 677, 219

Le Fèvre, O., Vettolani, G., Paltani, S., et al. 2004, A\&A, 428, 1043

Lilly, S. J., Fevre, O. L., Renzini, A., et al. 2009, VizieR Online Data Catalog, 217, 20070

Masters, K., Maraston, C., Nichol, R. C., et al. 2011, MNRAS, 418, 1055

Mignoli, M., Cimatti, A., Zamorani, G., et al. 2005, A\&A, 437, 883

Newman, J. A., Cooper, M. C., Davis, M., et al. 2012, arXiv:1203.3192

Norman, C., Hasinger, G., Giacconi, R., et al. 2002, ApJ, 571, 218

Pasquali, A., Ferreras, I., Panagia, N., et al. 2006, ApJ, 636, 115

Peng, C. Y., Ho, L. C., Impey, C. D., \& Rix, H. 2002, AJ, 124, 266

Pierce, C. M., Lotz, J. M., Primack, J. R., et al. 2010, MNRAS, 405, 718

Popesso, P., Dickinson, M., Nonino, M., et al. 2009, A\&A, 494, 443
Ravikumar, C. D., Puech, M., Flores, H., et al. 2007, A\&A, 465, 1099 Rhoads, J. E., Malhotra, S., Pirzkal, N., et al. 2009, ApJ, 697, 942 Rix, H.-W., Barden, M., Beckwith, S. V. W., et al. 2004, ApJS, 152, 163

Roche, N. D., Dunlop, J., Caputi, K. I., et al. 2006, MNRAS, 370, 74

Scoville, N., Abraham, R. G., Aussel, H., et al. 2007, ApJS, 172, 38

Simard, L. 1998, in ASP Conf. Ser. 145, Astronomical Data Analysis Software and Systems VII, ed. R. Albrecht, R. N. Hook, \& H. A. Bushouse (San Francisco, CA: ASP), 108

Straughn, A. N., Meurer, G. R., Pirzkal, N., et al. 2008, AJ, 135, 1624

Straughn, A. N., Pirzkal, N., Meurer, G. R., et al. 2009, AJ, 138, 1022

Strolger, L.-G., Riess, A. G., Dahlen, T., et al. 2004, ApJ, 613, 200

Szokoly, G. P., Bergeron, J., Hasinger, G., et al. 2004, ApJS, 155, 271

van der Wel, A., Franx, M., van Dokkum, P. G., et al. 2005, ApJ, 631, 145

Vanzella, E., Cristiani, S., Dickinson, M., et al. 2008, A\&A, 478, 83

Vanzella, E., Giavalisco, M., Dickinson, M., et al. 2009, ApJ, 695, 1163

Welikala, N., \& Kneib, J.-P. 2012, arXiv:1202.0494

Wirth, G. D., Willmer, C. N. A., Amico, P., et al. 2004, AJ, 127, 3121

Wolf, C., Hildebrandt, H., Taylor, E. N., \& Meisenheimer, K. 2008, A\&A, 492, 933

Wolf, C., Meisenheimer, K., Kleinheinrich, M., et al. 2004, A\&A, 421, 913

Wuyts, S., Labbé, I., Schreiber, N. M. F., et al. 2008, ApJ, 682, 985

Wuyts, S., van Dokkum, P. G., Franx, M., et al. 2009, ApJ, 706, 885 